\title{
Vertical-Edge-Based Car-License-Plate Detection Method
}

\author{
A.R.Isra ${ }^{1}$, A.Gokulanathan ${ }^{2}$ \\ ${ }^{I}$ Department of Electronics and Communication Engineering (Final Year) / Periyar Maniammai University, \\ Periyar Nagar, Vallam, Thanjavur-613403 Tamil Nadu, India . \\ ${ }^{2}$ M.E, Asst. Prof. /Department of Electronics and Communication Engineering) / Periyar Maniammai \\ University, Periyar Nagar,Vallam,Thanjavur-613403 Tamil Nadu, India .
}

\begin{abstract}
Vehicle plate recognition is a successful image processing technique used to recognize vehicles' plate numbers. There are several applications for this method which enlarge through many fields and attention groups. Vehicle plate recognition may be considered as an advertising equipment, for the purpose of traffic and border securities for law enforcement, and travel. Many methods have been accompanied to make this technique easy. This learning proposes an edge-detection method to allow a Plate Recognition System of a vehicle through the practical situations like the various environmental or meteorological conditions. Image processing tools are used to examine the plate area, resize it, and change it on the way to a gray scale earlier to filtering of the image in order to remove the unwanted areas. The obtained objects is processed in such a way that the number plate image and the information related to that is completely perfect The information of the obtained image is processed through the average deviation of the Gaussian filter (sigma).
\end{abstract}

Keywords: LPR, vehicle recognition, edge detection, access control, Gaussian filter

\section{Introduction}

The main application of digital image processing is the vehicle License Plate Recognition (LPR). This system is mainly accounted for the security and traffic installations purpose. The scientific conceptualization assumes the fact that the vehicles already have an identity proof displayed (the plate) and no additional transmitter or responder is required to be installed on the vehicle. The improvement of the LPR system is done to retain an image record of a vehicle for future references if needed in a case. In addition, this technology does not need any extra installation per vehicle. Other technological initiatives require a transmitter to be added on a vehicle or physically approved by the owner.Depending on the extracted plate number, the LPR system has a wide variety of applications and options to make the automated results for the problems. One of the uses is parking, in which the plate number is used to automatically enter pre-paid parks for members or determine parking fees for the non-members, the driver face recognition can be done to prevent car theft. The next is through access control, in which a gate by design opens for allowed members in a protected area, thus replacing the assistance of a security guard. In this case, when the gate opens. It can be made to log in a database based on the necessities. The third purpose is for tolling. The vehicle plate number is used to calculate a travel fee within a toll-road, or used to double-check the ticket. The fourth is for law enforcement use. The plate number is functional in arrange to produce a violation fine on speed or red-light systems. The fifth purpose is for the traffic organization where the vehicles moving in the different lane can be monitored and regulated.

Intelligent transport system (ITS) is a superior application in which the aim is to give the original services to the different modes of transport and traffic management works and enable various users to be comfortable, safe, and more synchronized, and 'smarter' use of transport networks. Traffic-flow measurement and automatic incident can be done using a video camera. Cameras are being installed on the transportation and traffic signals for monitoring the vehicles and to monitor breaking of traffic rules. Since video based detection systems such as those used in routine number plate recognition do not involve installing any mechanism straight into the road surface or roadbed, this type of system is known as a "non-intrusive" technique of traffic detection. Video captured from camera is fed into processors which analyze the changes in the video frames as the vehicles pass. The cameras are classically mounted on poles or structures above or adjacent to the roads to monitor.

\section{Related Works}

In [1] Sarfraz. M Ahmed. M.J et al presents Automatic license plate recognition systems (LPR) which helps to decrease the number of traffic violations and construct the streets safer. In that scheme they explained about anurbanized automatic scheme that locates Saudi license plates in a captured image regardless of the time and the license plate scale. The proposed system can stand slight tilting of the license plate. The localization process is complex due to the extreme varying nature of the background content.Good results were obtained using the localization stage. A second division of the system was developed for segmenting and distinguishing 
the characters in the positioned license plate. Locating the license plate in a picture is the first velocity in any license plate recognition system. In [2] Ozbay.S, and Ercelebi.E et al , the presentation is about the Automatic Vehicle Identification (AVI) which has a lot of applications in traffic systems (highway electronic toll collection, red light breach enforcement, edge and society checkpoints, etc.). License Plate Recognition is an effective form of AVI systems. In this study, a smart and simple algorithm is used for the vehicle's license plate recognition system. The planned algorithm consists of three main parts: Extraction of plate region, segmentation of characters and recognition of plate typescript. For extracting the plate district, edge detection algorithms and smearing algorithms are used. In segmentation part, smearing algorithms, filtering and some morphological algorithms are used. And statistical based pattern matching is done for recognition of plate characters. The performance of the proposed algorithm has been experienced on real images only.

In [3] Sulehria .H.K, Zhang.Ye et al ,the presentation is aboutAutomatic Identification of vehicles in a very difficult area, which is in disparity to the traditional practice of monitoring the vehicles physically. Automatic license plate (LP) recognition is one of the most aspects for the applying of computer apparition technique towards the intelligent transport system. In the Location of the vehicle plate, a method of vehicle license plate character segmentation and withdrawal based on improved edge detection and Mathematical morphology were accessible through the technology. Firstly, the color images were distorted into grey images. Secondly, the calculation of the dissimilarity of each pixel and neighborhood pixels is done to build up images edge and then it can be created as the license plate. Sobel operator is used to remove the edge of substance in image; then the algorithm applies the dilation forward and erosion mathematical morphology of binary images is done to get the image level contour.In [4] Chen.Z.X, Liu.Ch.Y et al the presentation is about theLicense-plate recognition which plays a significant role in numerous applications, and a number of techniques have been proposed. Here, a novel technique to distinguish license plates is done. Firstly, the license plates are located using the most important features. Then, each of the seven fonts in a license plate are segmented. Finally, the character recognizer extracts some of the most important skin of the characters and uses a feature salience classifier to achieve the robust recognition results. In the experiments, 1176 images that were in use from various scenes and circumstances were used, and 32 images out of the 1176 images failed to properly locate the license plates, which amounts for the achievement speed of $97.3 \%$. In these experiments on identifying the license characters, 1144 images were used for which license plates have successfully been located and out of which 49 images unsuccessful to classify the characters; the rate of successful identification is $95.7 \%$.

In [5] Suri. Dr. P.K, VermaEr. A et al the presentation is about the numbers on vehicle plates which is used as the input image and simple color conversion are used. Edge detection and connector measurement methods are also accounted too. Throughout the whole work, they use the masking and smoothing operations. The median filter is worn as one of the operators. The best consequences can be obtained by receiving the value of connector mechanism which is more than 17. Various methods have been planned so far but there is always a difficulty to notice the numbers. The image is stored in the form of a matrix and the output is displayed in the shape of detected numbers. They use Sobel Edge Detection technique .To minimize the complexity (as in covariance matrix) and local variance scores have been used and the sole coefficients have been efficient into a attribute vector form and multi-layer neural network. Since no explicit comparison or detachment calculation is essential in this framework, it is very easily possible to maintain the computational weight of the detection process low always.

\section{Existing Algorithms}

Various license plate (VLP) detection algorithms have been well developed in the past some years. These algorithms include both the advantages and disadvantages. And the majority of them use gradient edge detection.The most common approaches are VLP detection include texture, color feature, edge extraction, combining edge and color, morphological operation and learning-based method. The proposal in which license plate extraction had been done using Sobel filter, morphological operations and connected component scrutiny.The scheme for License Plate Detection and Segmentation for the Vehicles is based on the morphological operation like opening, dilation, erosion followed with filling. To extract the real license plate from image the centroid of the area is marked and depending on this license plate is finally extracted.In general, License plate location algorithm consists of steps like as Edge Detection, Morphological process similar to dilation and erosion, smoothing, segmentation of characters and recognition of plate characters is described. Here, they have implemented VLP based on canny edge detection. This approach is vigorous and simplest of the existing technique as far as completing time is troubled.

\section{Image Preprocessing}

The vehicle image is in low-quality along with the angle, lighting, weather etc. In this process, preprocessing the picture to capture the main information of plate is done.Frequently, the vehicle image preprocessing includes five steps: grayscale image; image smoothing; image sharpening; Binarization and edge 
detection of license plate. The preprocessing of vehicle image can reduce the difficulty of the original image in sequence, locate the license plate simply and fastly, make information clearly. Here, the analysis based on the edge detection is done.

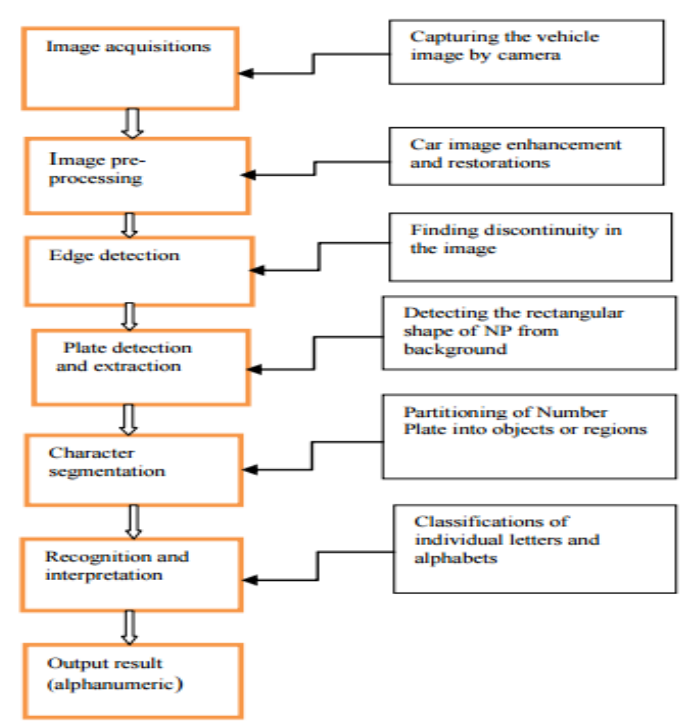

Fig 4.1 Overview of the Process

\section{Edge Detection}

In this recognition, edge detection is crucial to image preprocessing. Edge is a significant part in image; we can get some useful information's form it. It refers to some pixels with gray value suddenly changed and is collected of a figure of straight or bowed lines in image. The vehicle image obtained from various environments, so that it may contain diverse informations, such as passer, animal, grass, tree and so on. Although the setting of image is complex, the inherent edge character in license plate is clear. The appearance of plate is a rectangular, the height width ratio of it is a fixed value, and characters approved horizontally in the rectangular. The size of license plate in china is $440 \mathrm{~cm} \times 140 \mathrm{~cm}$ (length $\times$ width), and the tallness width ratio is about $3: 1$. There are 7 characters in the license plate, each character is $90 \mathrm{~cm} \times 45 \mathrm{~cm}$, the spacing of edge and character is $10 \mathrm{~cm}$, the spacing of each character is $12 \mathrm{~cm}$. Some gray values distorted in the license plate and background, characters and plate, these rich characteristics of edge represent a lot of high-frequency apparatus in the vehicle image. Rich edge informations can be taken to get the area of license plate from complex image with two steps: one is edge detection for images; the other is segmentation for the plate with inbuilt edge informations.There are many algorithms on edge detection, such as classical operator, multi-scale method and adaptive filtering process. Normally, each method is fitted for diverse container. We take a brief preamble in traditional Sobel and Prewitt operator in 2-2-1 and 2-2-2. These classic operators have strong ability of denoising, but the cost of reckoning is large.The use of these operator to process the vehicle images with multifaceted environmentwill result in a number of false edges. To resolve this problem, a novel adaptive edge detection algorithm based on first-order dissimilarity operator is done to notice the gray edge in vehicle image.

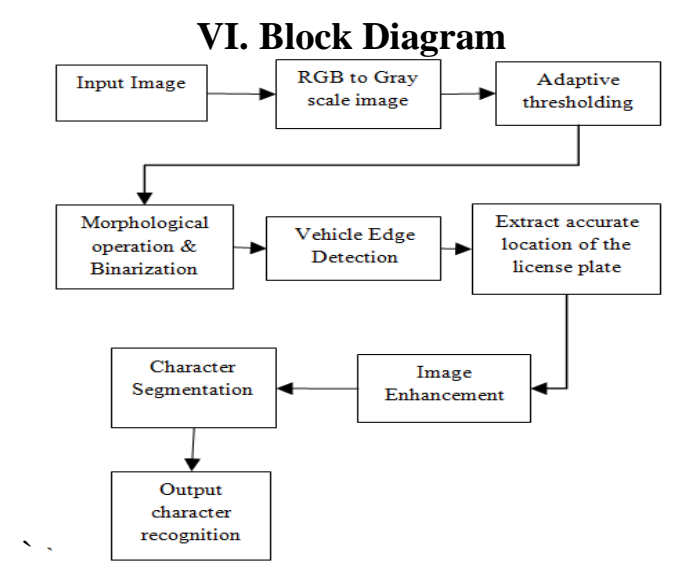

Fig 6.1 Block Diagram 


\section{Canny Edge Detection}

Edge-detection is an essential tool that is broadly used within image processing. It is functional practically in applications such as object determination in which feature detection aims to classify the certain objects of an image. Several edge-detection methods are widely based on the several possible optimization techniques. For instance, error minimization, maximizing an object function, fuzzy logic, wavelet advance, morphology, genetic algorithms, neural network and Bayesian approach. Various edge-detection methods perform to wavering degrees of quality within distorted surroundings. Therefore, it is probable to apply numerous edge-detection algorithms.

The Canny Edge-Detector is an operator which uses a multi-stage algorithm to decide a wide variety of edges in a noisy illustration as follows

1 .Use the Gaussian filter $G \sigma(\mathrm{m}, \mathrm{n})$ to smooth out the image $\mathrm{f}(\mathrm{m}, \mathrm{n})$. This will reduce noise or unwanted details and textures as given in Eq.(1).

$\mathrm{g}(\mathrm{m}, \mathrm{n})=G \sigma(\mathrm{m}, \mathrm{n}) * \mathrm{f}(\mathrm{m}, \mathrm{n})$

Where $G \sigma(\mathrm{m}, \mathrm{n})$ is given as

$G \sigma(m, n)=\frac{1}{\sqrt{2 \pi \sigma^{2}}} \exp \left[-\frac{m^{2}+n^{2}}{2 \sigma^{2}}\right]$.

2. Compute gradient of $\mathrm{g}(\mathrm{m}, \mathrm{n})$ using any of the gradient operators to reach

$$
M(m, n)=\sqrt{g_{m}^{2}(m, n)+g_{n}^{2}(m, n)} .
$$

3. Threshold $\mathrm{M}$ is given as:

$M_{T}(m, n)=\left\{\begin{array}{cc}M(m, n) & \text { if } M(m, n)>T \\ 0 & \text { otherwise }\end{array} .\right.$.

Where $\mathrm{T}$ is taken such that all edge elements are kept and most of the noise is suppressed.

4. Suppress non-maxima pixels in the edges of MT are obtained above to thin the edge ridges. Check whether each non-zero MT $(m, n)$ is greater than its two neighbors along the gradient direction $\theta(m, n)$. If so, keep MT $(\mathrm{m}, \mathrm{n})$ unchanged, otherwise, set it to zero.

5. Threshold the previous result by two different thresholds $\tau 1$ and $\tau 2$ (where $\tau 1<\tau 2$ ) to obtain two binary images T1 and T2. This T2 has less noise and fewer false edges than T1 but it also has more gaps between those edge segments.

6. Link edges segments in $\mathrm{T} 2$ to form continuous edges. To do so, trace each segment in $\mathrm{T} 2$ to its end and then search its neighbors in $\mathrm{T} 1$ to find any edge segment in T1 until it reaches another edge segment in $\mathrm{T} 2$.

\section{Plate Number Recognition Algorithm}

The main purposes of this is to investigate the ability of capturing and recognizing plate information of vehicles using a correctly permanent camera. The image will be kept for additional processing or it may be compared against specific databases, such that if a plate number matches to the one already existing, then the system is enabled. The algorithm is based on clear and easy image processing tools and is summarized as follows:

1. Read and resize the image

2. Convert the image toward a gray scale

3. Receive the complement of the image and find the edges

4. Filter the image information of small objects.

5. Segregate the image into objects

6. Recognise the license plate

Each step of the algorithm process has its role, priority, and contribution in the direction of having a comprehensive algorithm. Reading the overall image is a preliminary point in order to construct the general impression of the plate itself. Resizing the image is done. Several edge-detection approaches may be used to obtain the plate of a vehicle. The edge-detection process leads to identifying different objects within an image. Therefore, using suitable filters, unwanted objects are easily removed and essential objects of the image remain. These objects are separated in such a way that a plate is familiar, recognized, and acquainted to its suitable place. 


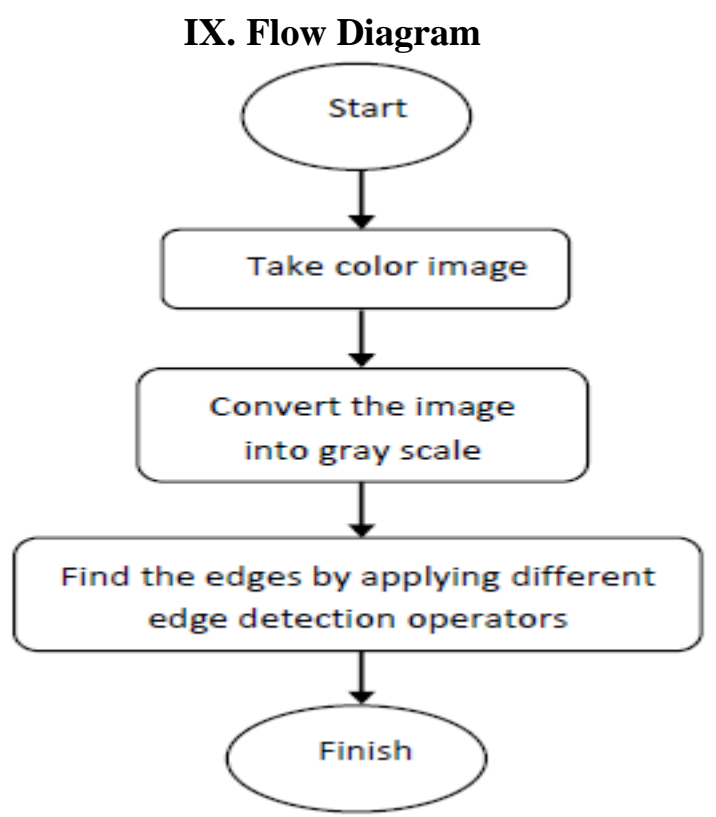

Fig 9.1 Flow Diagram

\subsection{Read and resize the image}

\section{Proposed Process}

Take the picture of the license plate area in use and the image is resized into a suitable ratio. These values are selected so that the width of the image is 600 pixels and the length is 400 pixels as illustrated.

\subsection{Convert the image toward gray scale}

The image is smoothed by its alteration near gray scale, enabling the coordination to locate the edges of the image. This gray scale image is improved to facilitate the routine of the system.

\subsection{Receive the complement of the image and find the edges}

The complement of the image is obtained and is improved again. An edge-detection method is used to obtain the edges of the mandatory image for further actions. Canny Edge-Detection method has been used to find such values of the troubled image based on local maxima of the gradient of that image. The gradient is considered using the derivative of a Gaussian filter. This method uses two thresholds to distinguish strong and weak edges and it considers the standard deviation of the Gaussian filter (sigma). This factor controls the amount of particulars of an image to be filtered. This is so when sigma increases, fewer details of the filtered image stay. The value of sigma has been selected as 0.95 which proves to be a more than adequate option in obtaining fair consequences as illustrated

10.4 Filter the image of small objects

The image is filtered and a number of objects are formed with different sizes. Small size objects have been removed. This is intended by if the size of an object is fewer than a predefined threshold price. This value has been set to 280 pixels and so the outstanding objects are measured as illustrated.

10.5 Separate the image into objects

Objects are estranged and each object has a specific feature that enables the scheme to know the plate-like object as shown in Figure. This step is necessary in simplifying the outstanding processes based on a variety of separated objects inside the image.

\section{Simulation Result}

In order to recognize the license plate from those objects, an image consisting of objects is preferred. A suitable explanation of the license plate is based on solidity, the ratio among the height and width, convex area, and bounding box. Accordingly, the plate can be acknowledged by multiplying the plate object with the original image as illustrated

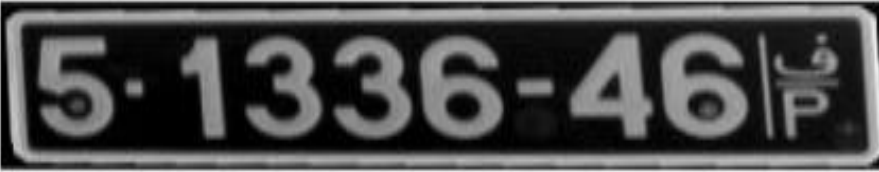

Fig 11.1 Stimulated Output 


\section{Conclusion}

Vehicle LPR has more than a few applications. It also has several approaches and algorithms. Practical image processing tools have been used to enable the system in order to obtain the desired objects and so to identify license plate numbers. This proposed advance enables the coordination to zoom smoothly toward the exact frame of the plate.Superfluous objects are simply detached and the license plate is standard.

\section{References}

[1]. M. Rasooli, S. Ghofrani and E. Fatemizadeh, "Farsi License Plate Detection based on Element Analysis and Characters Recognition”, International Journal of Signal Processing, Image Processing and Pattern Recognition, vol. 4, no. 1, (2011) March, pp. $65-80$.

[2]. M. M. Shidore and S. P. Narote, "Number Plate Recognition for Indian Vehicles", IJCSNS International Journal of Computer Science and Network Security, vol. 11, no. 2, (2011) February, pp. 143-146.

[3]. B. Shan, "Vehicle License Plate Recognition Based on Text-line Construction and Multilevel RBF", Journal of Computers, vol. 6, no. 2, (2011) February, pp. 246-253.

[4]. S. Ozbay and E. Ercelebi, "Automatic Vehicle Identification by Plate Recognition A Neural Network", World Academy of Science, Engineering and Technology, vol. 9, (2005), pp. 222-225.

[5]. M. I. Khalil, "Car Plate Recognition Using the Template Matching Method", International Journal of Computer Theory and Engineering, vol. 2, no. 5, (2010) October, pp. 683-687.

[6]. Z. Shaaban, "An Intelligent License Plate Recognition system", International Journal of Computer Science and Network Security, vol. 11, no. 7, (2011) July, pp. 55-61.

[7]. O. Khalifa, S. Khan, R. Islam and A. Suleiman, "Malaysian Vehicle License Plate Recognition", The International Arabournal of Information Technology (IAJIT), vol. 4, no. 4, (2007) October, pp. 359-364.

[8]. Y. Liu, L. Cui, J. Shu and G. Xin, "License Plate Location Method Based on Binary Image Jump and Mathematical Morphology", International Journal of Digital Content Technology and its Applications, vol. 5, no. 5, (2011) May, pp. 259-265.

[9]. Z. Shaaban, "An Intelligent License Plate Recognition System”, IJCSNS International Journal of Computer Science and Network Security, vol. 11, no. 7, (2011) July, pp. 55-61.

[10]. R. Maini and H. Aggarwal, "Study and Comparison of Various Image Edge Detection Techniques", International Journal of Image Processing (IJIP), vol. 3, Issue 1, (2009) February, pp. 1-12.

[11]. P. Bao, L. Zhang and X. Wu, "Canny edge detection enhancement by scale multiplication", IEEE Transactions on Pattern Analysis and Machine Intelligence, vol. 27, Issue 9, (2005) September, pp. 1485- 1490.

[12]. K. Parasuraman, "SVM Based License Plate Recognition System", 2010 IEEE International Conference on Computational Intelligence and Computing Research, (2010) December 28-29, India

[13]. Y. M. Alginahi, “Automatic Arabic License Plate Recognition”, International Journal of Computer and Electrical Engineering, vol 3, no. 3, (2011) June, pp. 454-460.

[14]. A. P. Nagare, "License Plate Character Recognition System using Neural Network", International Journal of Computer Applications, vol. 25, no. 10, (2011) July, pp. 36-39. 